\title{
Delta-1-Pyrroline-5-Carboxylate Dehydrogenase, Mitochondrial
}

National Cancer Institute

\section{Source}

National Cancer Institute. Delta-1-Pyrroline-5-Carboxylate Dehydrogenase,

Mitochondrial. NCI Thesaurus. Code C152045.

Delta-1-pyrroline-5-carboxylate dehydrogenase, mitochondrial (563 aa, $62 \mathrm{kDa}$ ) is encoded by the human ALDH4A1 gene. This protein is involved in the conversion of proline pyrroline-5-carboxylate to glutamate. 